\title{
Prospectives of the Hadron Program in ATLAS
}

\author{
R. Kwee ${ }^{1,2)}$ on behalf of the ATLAS Collaboration \\ 1 (CERN, 1211 Geneve 23, Switzerland) \\ 2 (Humboldt University of Berlin: Physics Department, Experimental Particle Physics, 12489 Berlin, Germany)
}

\begin{abstract}
One of the first measurements that will be made at the LHC by ATLAS deals with the properties of inelastic collisions, namely the central charged particle density and transverse momentum distributions. Current predictions of these distributions have large uncertainties in the LHC energy range. We describe the ATLAS minimum bias triggers, designed to select all kind of inelastic interactions, and the performance of the track reconstruction software which was adapted to soft particle track reconstruction. The precision with which the minimum bias distributions can be measured with early data is presented and the uncertainties on the inelastic distributions due to trigger bias is discussed.
\end{abstract}

Key words ATLAS, LHC, Minimum Bias, Minimum Bias Trigger, Underlying Event, early measurements

PACS 13.85.Hd Inelastic scattering: many-particle final states

\section{Introduction}

ATLAS is one of the four major experiments at the Large Hadron Collider (LHC). The machine operation has successfully re-started in November 2009, providing proton-proton collisions to the four experiments. A wide range of physics analyses is envisaged to exploit the physics potential when the accelerator is operated at its nominal parameters of energy $\sqrt{s}=$ $14 \mathrm{TeV}$ and high luminosities of $\mathrm{L}=10^{34} \mathrm{~cm}^{-2} \mathrm{~s}^{-1}$. Searches for Higgs bosons of masses up to the electroweak energy scale of $1 \mathrm{TeV}$, possible deviations from the Standard Model and also unknown signatures are the objectives of the ATLAS physics program. Their studies and expectations can be found in ref. ${ }^{[1]}$. However, ATLAS has the potential already at initial lower energies of $\sqrt{s}=7$ and $10 \mathrm{TeV}$ and luminosities of $\mathrm{L}=10^{31} \mathrm{~cm}^{-2} \mathrm{~s}^{-1}$, to improve the knowledge of the inelastic scattering processes and of the multi-parton interactions by performing measurements of interactions with a small momentum transfer.

Minimum Bias (MB) and Underlying Event (UE) analyses are the main hadron studies of soft interactions with early data. In both cases the motivation is that soft interaction processes are yet not well understood. It is therefore important to quantify the properties of the soft processes at all available center of mass energies. The best currently available de- scriptions come from phenomenological models where multiple parton interaction is one of the key ingredients to simulate charged particle multiplicities. With data, we will be able to improve the descriptions, which are used in Monte-Carlo event generators like Pythia $^{[6]}$ or Phojet ${ }^{[7]}$. The ultimate goal is to describe soft interactions in the fundamental language of QCD. Results of the analyses of soft processes will have a crucial impact on all analysis of high- $p_{T}$ processes, where e.g. the determination of the jet energy and missing transverse energy scale is needed. Also lepton isolation or the direct Higgs-search in the vector-boson-fusion channel require the understanding of low- $p_{T}$ QCD analysis beforehand.

This paper focuses on early measurements of the properties of inelastic interactions. In particular, the studies of the kinematic spectra of charged particle distributions $d N_{c h} / d p_{T}$ and $d N_{c h} / d \eta$ are presented.

\section{Minimum Bias Analysis}

Events associated with MB physics comprise contributions from inelastic interactions. They can be furthermore classified as events with diffractive and non-diffractive dissociation of the nucleon. The diffractive dissociation, short diffractives, can be subdivided into single and double diffractives (sd, dd). These events are characterized by the presence of the

Received December 2009

1) E-mail:rkwee@mail.cern.ch

(C)2009 Chinese Physical Society and the Institute of High Energy Physics of the Chinese Academy of Sciences and the Institute of Modern Physics of the Chinese Academy of Sciences and IOP Publishing Ltd 
so called rapidity gaps which are regions, where final state particles are exponentially suppressed. In contrast, in a non-diffractive (nd) process a color field over the whole range in rapidity is formed allowing for mostly soft particle production. In former MB analyses e.g. made by the $\mathrm{CDF}$ collaboration ${ }^{[5]} \mathrm{MB}$ events were only associated to non-single diffractive events, excluding double-diffractive events. The exact definition of $\mathrm{MB}$ events is given by the trigger system. In ATLAS, the MB trigger systems are sensitive to all kinds of diffractive events, in addition to the non-diffractive processes. To compare also to previous results, ATLAS will measure also the non-single-diffractive distributions. These studies are shown here.

\subsection{Monte Carlo Predictions}

Two different Monte Carlo generators were used to compare predictions of cross-sections and kinematic spectra of charged particles, Pythia 6.420 and Phojet 1.12. They have a different way of modelling the soft QCD processes: while Pythia uses an approach combining Regge theory and an eikonalized QCD model, the dual parton model is implemented in Phojet. The cross-sections for $\sqrt{s}=10$ and 14 $\mathrm{TeV}$ are shown in Tab. 1 for the single inelastic processes. One can clearly see significant differences, which reach about $50 \%$ for (dd) events.

A similar difference is also visible in the kinematic spectra of charged stable particles (shown here for $\sqrt{s}=14 \mathrm{TeV}$ only). The distribution for pseudorapidity $^{*} \eta$ is shown in Fig. 1, for the transverse momentum $p_{T}$ distribution in Fig. 2. While Phojet seems to generate less charged particles per $\eta$ - and $p_{T}$-unit in the nd-events, the different modelling of the diffractive processes seems to be reflected especially in the shape of the $p_{T}$-distribution, showing a much larger tail to higher $p_{T}$ values in Phojet generated events. Also a clear difference is visible for the diffractive events.

Table 1. Cross-section predictions for $\sqrt{s}=10 \mathrm{TeV}(14 \mathrm{TeV})$.

\begin{tabular}{ccc}
\hline$\sigma / \mathrm{mb}$ & Pythia 6.420 & Phojet 1.12 \\
\hline$\sigma_{\text {total }}$ & $96(101)$ & $113(119)$ \\
$\sigma_{\text {inel }}$ & $75(79)$ & $81(84)$ \\
$\sigma_{\text {nd }}$ & $52(55)$ & $65(68)$ \\
$\sigma_{\text {dd }}$ & $9.8(10.3)$ & $4.0(4.1)$ \\
$\sigma_{\text {sd }}$ & $14.0(14.3)$ & $10.9(11.0)$ \\
\hline
\end{tabular}

${ }^{*} \eta=-\ln (\tan \theta / 2)$, where $\theta$ is the polar angle

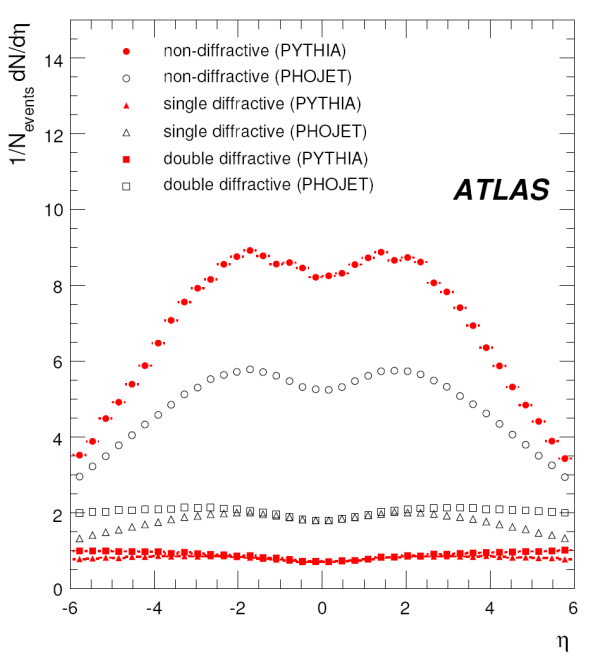

Fig. 1. Pseudorapidity distribution of charged particles from Pythia 6.420 and Phojet 1.12 shown for different inelastic processes, see ref. [1].

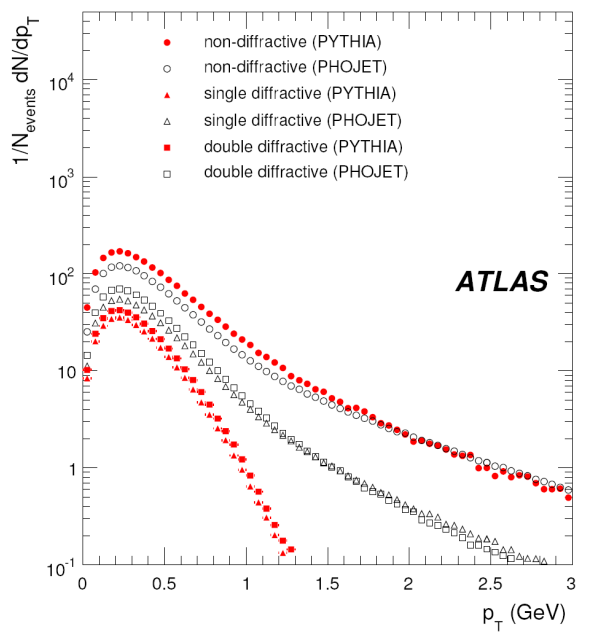

Fig. 2. Transverse momentum distribution of charged particles from Pythia 6.420 and Phojet 1.12 shown for different inelastic processes, see ref. [1].

\subsection{Analysis Procedure}

ATLAS will perform these measurements right from the beginning of data-taking, making use of minimum bias triggers. With increasing luminosities, a zero bias trigger will replace them. Several factors have to be considered in order to correct for inefficiencies caused by the trigger and track reconstruction. The systematic uncertainties need to be estimated. The procedure had been studied with $\sqrt{s}=14 \mathrm{TeV}$ $\mathrm{MC}$ data and is outlined in the following sections. Full details are in ref. ${ }^{[1]}$.

\subsection{Minimum Bias Trigger}

ATLAS possesses two main independent minimum bias trigger systems, both optimized to effi- 
ciently suppress empty bunch-crossing events, as they form the main background source at low luminosities, while triggering efficiently on inelastic interactions. One of such systems consists of Minimum Bias Trigger Scintillators (MBTS) that is made up of two detector parts with 16 scintillator modules, belonging to the first trigger level (L1) trigger ${ }^{\dagger}$. They are situated at about $3.5 \mathrm{~m}$ away from the interaction point (IP) on each side of the ATLAS detector and cover an $\eta$ range of $2.1<|\eta|<3.8$.

An alternative approach is realised by the Inner Detector (ID) Minimum Bias Trigger, covering the ID range of $|\eta|<2.5$, which is the region where track based measurements are performed. This trigger is seeded by a random trigger at L1 to minimize inital bias in the event selection. The actual event selection takes place at the high-level trigger (HLT), where in two trigger levels signals of the pixel and semiconductor tracker (SCT) detectors ${ }^{[3]}$ are required. At the second trigger level (L2) silicon hits above electronic noise are required, while at the third trigger level, the Event Filter (EF), a track requirement is imposed, such that tracks close to the nominal IP have to be found.

The trigger efficiency, here defined as the ratio of events with and without trigger requirement, is shown as a function of the trigger threshold: for MBTS the L1 requirement to have a multiplicity of 2 hits in the MBTS counters in Fig. 3 and for the ID Minimum Bias trigger passing the L2 silicon hit requirement in Fig. 4.

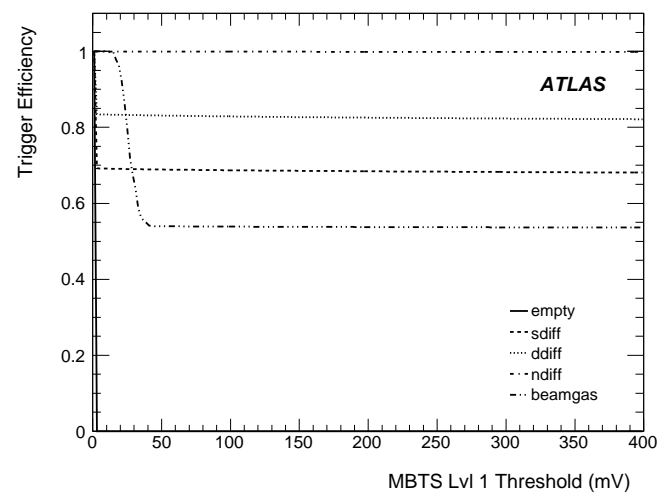

Fig. 3. MBTS trigger efficiency with a 2-hit multiplicity requirement for signal (inelastic processes) and background (empty events, beamgas events). Already at a very low threshold the empty events are completely suppressed.

\footnotetext{
${ }^{\dagger}$ For details of the ATLAS trigger system, see ${ }^{[2]}$.
}

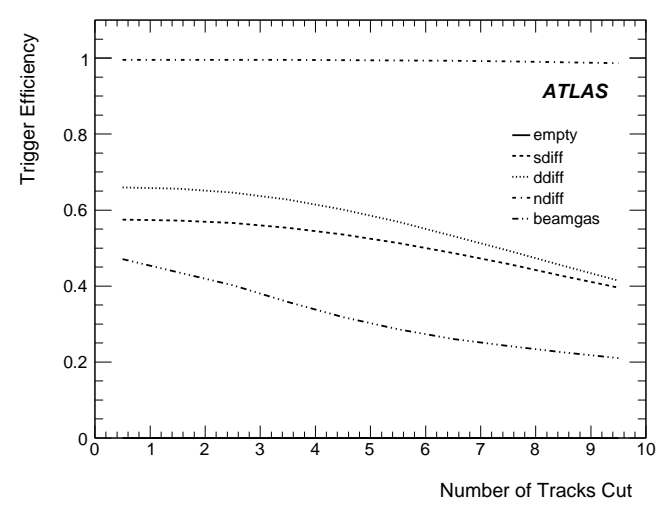

Fig. 4. ID Minimum Bias Trigger efficiency as a function of a track multiplicity requirement.

\subsection{Event and Track Selection}

For the analysis, the events were selected by the L1 MBTS trigger with the 2-hit multiplicity requirement. Additionally, offline selection criteria were applied, such that only events containing a reconstructed primary vertex and tracks with a $p_{T} \geq 150$ $\mathrm{MeV}$ satisfying quality criteria were considered in the analysis.

\subsection{Corrections}

The selection criteria imply that corrections have to be taken into account. The complexity arises as dependencies exist amongst various requirements. For the measurement of $d N_{c h} / d p_{T}$ and $d N_{c h} / d \eta$, the corrections account for trigger bias in the event selection and track and vertex reconstruction efficiencies. At event-level the corrections for trigger bias were calculated by relating the number of events of a particular inelastic processed to the number of events selected by the trigger. For this study, the bias correction is model-dependent, since it takes into account the relative cross-sections of the different inelastic physics processes. The vertex correction accounts for inefficiency of the vertex reconstruction, to not reconstruct primary vertex, even though charged particles were generated in the measured region.

A track-to-particle correction at track-level considers the track reconstruction efficiency, when generated charged primary particles were not reconstructed or fake and secondary tracks contribute to the primary tracks. The tracking efficiency is defined as the number of reconstructed primary tracks matched to the generated track particle over the number of generated charged primary particles, and is shown as function of $p_{T}$ in Fig. 5, where the default reconstruction cut has been lowered to $p_{T}=100 \mathrm{MeV}$. 


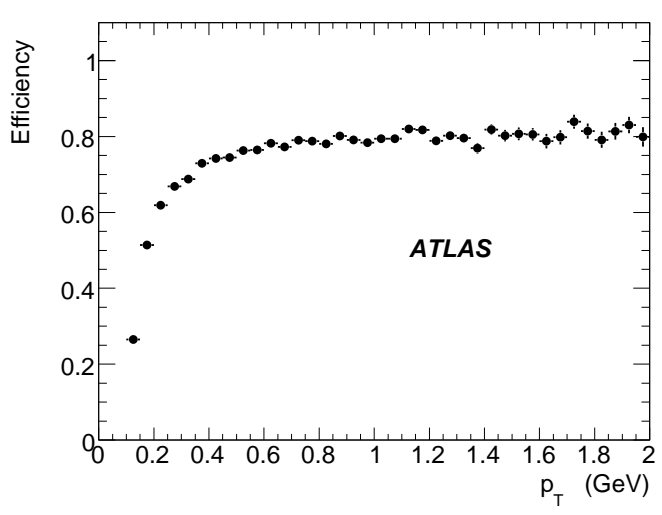

Fig. 5. Tracking efficiency as a function of $p_{T}$ in $|\eta|<2.5$

The result for the track-to-particle corrections is a function of $\eta, p_{T}$ and the number of charged particles $N_{c h}$, here shown projected to the $\eta$-axis in Fig. 6 . One can see, that the corrections are smaller in the central part of $|\eta|<1$.

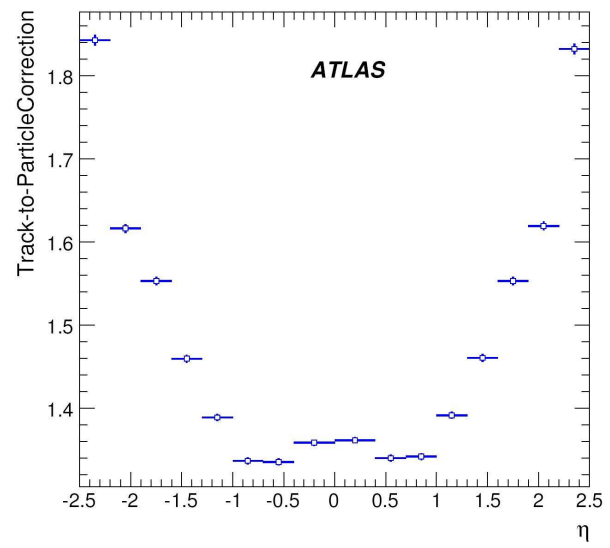

Fig. 6. Track-to-particle corrections as a function of $\eta$.

Systematic uncertainties were evaluated by changing parameters in the particle generation and reconstruction and re-calculating the corrections. It was found that the major source of systematic uncertainties comes from the diffractive cross-section $\sim 4 \%$. The results of the studies confirm that this procedure is able to reproduce the $d N_{c h} / d p_{T}, d N_{c h} / d \eta$ distributions which agree well with the Pythia MC predicted distributions.

\section{Underlying Event}

The activity of the UE manifests itself in additional production of mostly soft particles: similar to MB physics, the study of the UE also deals with multiple parton interactions and soft hadronic particle production. Accordnig to the common definition, the UE comprises all particles accompanying the hard scattering component of the collision. However, experimentally this separation is not possible. There- fore, topological regions are defined which are sensitive to the UE activity. Based on the direction of the leading jet or track $p_{T}$, the plane orthogonal to the beam direction is divided into 3 regions: the forward, away and transverse region. The transverse area is sensitive to the UE activity and typical observables in that region are e.g. the charged particle density, $p_{T}$, mean $p_{T}$ and scalar sum of $p_{T}$ of tracks and jets. As example, the mean multiplicity of charged particles in the transverse region is shown in Fig. 7, where it is clearly visible that also for the UE activity the preditions are highly uncertain ${ }^{[8]}$.

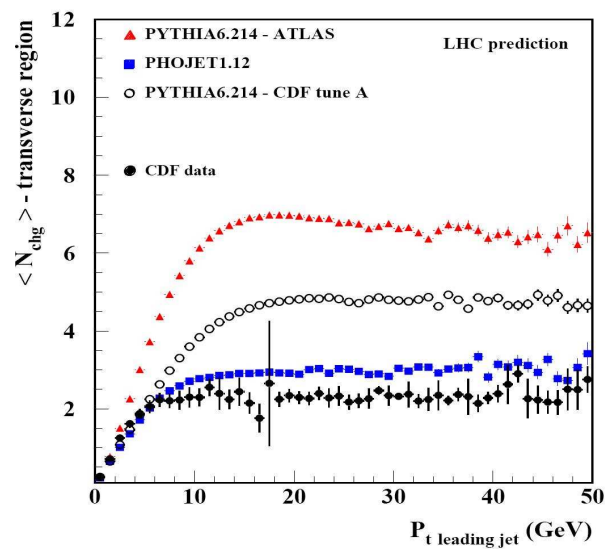

Fig. 7. MC predictions of UE activity for LHC energies. Different Pythia tunes and both MC generators show distinct activity levels in the mean multiplicity of charged particles.

\subsection{Analysis Goals}

Measuring the UE activity will help to distinguish different physics models. An inital beam energy of $\sqrt{s}=7 \mathrm{TeV}$ and an integrated luminosity of $10 \mathrm{pb}^{-1}$ are already useful for such studies. Nevertheless the systematic uncertainties need to be understood first.

\section{Conclusion}

The hadron program of ATLAS starts with the very first measurements of the properties of the inelastic interactions, which will help understanding soft QCD models. Additional hadron analyses are described in ref. ${ }^{[4]}$.

\section{References}

1 The ATLAS Collaboration, Expected Performance of the ATLAS Experiment, Detector, Trigger and Physics, CERN-OPEN-2008-020, Dec. 2008 Technical Design Report, ATLAS High-Level Trigger Data Aquisition and Controls, CERN/LHCC/2003-22, ATLAS TDR 016, 30 June 2003

Controls, CERN/LHCC/2003-22, ATLAS TDR 016, 30 June 2003
Inner Detector Technical Design Report, ATLAS TDR 5, Inner Detector Technical Design Report, ATLA
CERN/LHCC/97-17, ISBN 92-9083-103-0, 30. April 1997

CERN/LHCC/97-17, ISBN 92-9083-103-0, 30. April 1997
K. Hara for the ATLAS Collaboration, ATLAS commissioning and early K. Hara for the ATLAS Collaboration, ATLAS commission
physics of resonance and jet production, this conference.

5 The CDF Collaboration, Measurement of Particle Production and Inclusive differential cross-sections in $\mathrm{p} \bar{p}$ Collisions at $\sqrt{s}=1.96 \mathrm{TeV}$, arXiv:0904.1098 [hep-ex]

PYTHIA 6.400 manual, JHEP 05 (2006) 026

7 R. Engel, PHOJET manual (ver. 1.05c, June 1996)

8 A. Moraes, C. Buttar, D. Clements, Measuring the underlying event at ATLAS, CERN-ATL-PHYS-PUB-2005-015 